\title{
OAODV Routing Algorithm for Improving Energy Efficiency in MANET
}

\author{
Suvarna P. Bhatsangave \\ (IEEE Student Member) \\ ME - II Year, P. G. Department (Comp. \\ Networking and Engineering) \\ M. B. E. Society's College of Engineering, \\ Ambajogai
}

\author{
V. R. Chirchi \\ (IEEE Member) \\ Asst. Prof. in P. G. Department (Comp. \\ Networking and Engineering) \\ M. B. E. Society's College of Engineeering, \\ Ambajogai
}

\begin{abstract}
In mobile ad hoc networks (MANETs), there is no fixed infrastructure. One of the most widely used routing protocols for an ad hoc network is the Ad hoc On Demand Distance Vector routing protocol, abbreviated as AODV. In the conventional AODV routing protocol, source node forwards RREQ (Route Request) packet to find out path to the destination node. The intermediate node having less lifetime or energy, also forwards RREQ. As lifetime expires after some time i.e. node goes down; it could not forward RREP (Route Reply) on reverse path. Hence, source node has to restart RREQ rebroadcast to communicate with destination, which results in unnecessary RREQ rebroadcast, less Packet Delivery Ratio (PDR) as well as throughput and more end to end delay.
\end{abstract}

Solution to above problem is given in this paper, by Optimized AODV (OAODV) routing protocol. In this, the node does not forward RREQ unless there is sufficient energy (battery lifetime), and until the node density in its surrounding exceeds a particular threshold. These two parameters are defined taking into consideration various statistics. Optimized AODV analyzes these two parameters, when implementing routing discovery, and avoiding the unnecessary information sending efficiently. By comparing AODV with optimized AODV in the same scenario, the new protocol is much better than AODV in terms of battery lifetime and throughput.

\section{General Terms}

Ad hoc network routing protocol, Wireless ad hoc networks et. al.

\section{Keywords}

Energy efficient routing protocol, battery lifetime, throughput, QoS, OAODV.

\section{INTRODUCTION}

In infrastructureless wireless networks, there is no need to use a base station. Each node acts as a router. Conventional protocols of wired networks are not suited for ad hoc networks. Hence, there is a need to design new protocols to work in wireless medium [1]. MANETs are becoming popular across the globe because of simple deployment and less cost of IEEE 802.11 standard. Network members may be inside
PDA (Personal Digital Assistant), laptops, digital cameras, mobile phones, etc. [2].

\begin{abstract}
Mobile ad hoc networks consist of mobile nodes which operate on battery. A mobile node has a finite and decreasing energy. Therefore, these nodes need to be energy conserved to maximize the battery life. Energy management is the task of MAC (Medium Access Control) layer [3] while the network layer can take decisions based on topology or traffic characteristics. Energy consumed by the sleeping state node is significantly less than the transmit/receive/idle state node. To minimize energy consumption, path which consumes less power is also can be chosen [4].
\end{abstract}

For the last decade, many researches had been performed in mobile ad hoc networks (MANETs), especially in routing protocol of Ad hoc on demand Distance Vector (AODV) for the optimization or better performance [5].

\section{REVIEW OF AODV ROUTING PROTOCOL}

Wireless mobile ad hoc networks are characterized as networks without any physical connections. In these networks there is no fixed topology due to the mobility of nodes, interference, multipath propagation and path loss. Hence a dynamic routing protocol is needed for these networks to function properly [6]. Many routing protocols have been developed for accomplishing this task. The widely used routing protocols for MANETs are DSDV (Destination Sequenced Distance Vector), DSR (Dynamic Source Routing) and AODV [7].

In 1994, Charles Perkins [8] presented DSDV, which is a proactive routing protocol. It is a modification of Bellman Ford mechanism. In this protocol, source node always has a path to destination in the form of route table at all times i.e. paths are available the moment they are needed. DSDV advertises periodic and event triggered advertisements throughout the network whenever there is a change in topology. Each node changes its sequence number after receiving updates. The node having greatest sequence number is chosen. Each node is having IP address of source and destination, current sequence number and hop count in its route table. The node removes stale entries from route table to guarantee loop problem. System wide updates consume some amount of battery and bandwidth, even if the network is idle. So, DSDV is not suitable for highly dynamic networks. 
In 1996, David Johnson and David Maltz [9] proposed DSR which is a reactive routing protocol. Unlike DSDV, DSR starts path finding process only when there is a demand. Source routes are carried out in each data packet. Two mechanisms are involved i.e. route discovery and maintenance.

In the early 2000s, researchers focused on the development of basic functions or services of the AODV protocol, such as shared channel, route discovery, and dynamic nodes. The purpose of their studies was to manage an ad hoc network topology that always change and answer the problem of disconnected route (route error) caused by the level of mobility [10].

In 2001, C. E. Perkins, E. M. Royer and S. Das [11] proposed Ad hoc On Demand Distance Vector (AODV) routing protocol which functions similar to DSR protocol. But, instead of carrying out source routes in each packet as in DSR, AODV maintains route table entries at intermediate nodes. AODV also maintains destination sequence number to avoid loop problem. AODV works efficiently for large number of nodes which is not the case for DSDV.

This paper tells that, reducing power consumption and efficient battery life of nodes in an ad hoc network requires an integrated power control and routing strategy. The power control is achieved by new route selection mechanisms for MANET routing protocols. In 2005, K. Murugan and S. Shanmugavel [12] proposed Energy Based Time Delay Routing (EBTDR) and Highest Energy Routing (HER). These algorithms try to increase the operational lifetime of an ad hoc network by implementing a couple of modifications to the basic DSR protocol and making it energy efficient in routing packets. The modification in EBTDR is such that if the nodes' remaining energy is less, then packets are forwarded after some time i.e. delay is introduced. If nodes' remaining energy is high then packets are forwarded immediately i.e. there is no concern of delay. In HER, the route selection is based on the energy drain rate information in the route request packet. It is observed from the simulation results that the proposed algorithms increase the lifetime of mobile ad hoc networks, at the expense of system complexity and realization.

In 2008, Thriveni and et al. [13] proposed an algorithm to improve the flooding performance of an Ad Hoc on Demand Distance Vector (AODV) routing protocol called, Probabilistic Mean Energy Flooding (PMEF) which periodically performs an averaging. As the word Mean Energy is there, algorithm calculates average energy say $\mathrm{E}_{\text {avg. }}$. Remaining energy is also calculated called $\mathrm{E}_{\mathrm{r} \text {. Route selection }}$ depends on the probability which is drawn on the basis of difference between residual energy $E_{r}$ and mean energy $E_{\text {avg. }}$. This algorithm is used in route discovery process to make a rebroadcast decision by the node. If, nodes does not have sufficient energy, then rebroadcasting of packet is not done. As compared to the existing AODV, proposed schemes in forwarding a route request are more effective in reducing the flooding overhead and increase the network lifetime and throughput thereby decreasing the network latency.

In 2009, Zhang Jianwu, Zou Jingyuan and Zhao Qi [14], proposed modifications to improve the broadcasting mechanism of AODV protocol. They presented an improved mobile ad hoc network on demand routing protocol which is based on AODV. It controls the broadcasting of RREQ information. This protocol analyzes the lifetime of node, when implementing routing discovery, and avoiding the unnecessary information sending efficiently. By comparing AODV with OAODV in the same scenario, the new protocol is much better than AODV in terms of packet delivery ratio as well as routing load.

In 2011, Sunil Taneja and et al. [15] proposed a scheme that takes into consideration power status of each and every node in the topology and further ensures the fast selection of routes with minimal efforts and faster recovery. Battery strength of nodes is divided into three states namely danger state, critical state and active state. The nodes which are in active state participate in route selection. The results have been derived by carrying out experiments over network simulator NS-2. The performance evaluation of new AODV and existing AODV has been done on the basis of packet delivery ratio and exhausted nodes. The proposed scheme in new AODV works on a reactive approach and utilizes alternate paths by satisfying a set of energy based criteria. This scheme can be incorporated into any ad hoc on demand routing protocol to reduce frequent route discoveries. Alternate routes are utilized only when data cannot be delivered through the primary route. Simulation results indicate that the proposed scheme provides robustness to mobility and enhances protocol performance. Average increases in terms of packet delivery ratio for different network scenarios.

\section{AODV OPERATION}

This section describes the scenarios under which nodes generate Route Request (RREQ), Route Reply (RREP) and Route Error (RERR) messages.

\subsection{AODV Route Discovery}

When source node wants to communicate with destination and if path is not available to destination then source floods or broadcasts RREQ i.e. request packet to all its neighbours in the network. This RREQ message contains source and destination node's IP address, sequence number of destination, its current sequence number, hop count and RREQ ID. RREQ ID is monotonically increasing number. It gets incremented after each node initiates new RREQ. Figure 1 illustrates this flooding procedure [16].

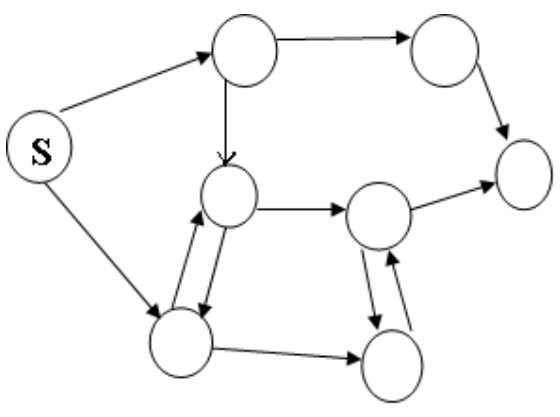

Fig 1: RREQ Broadcast [17].

When intermediate node receives RREQ, they create reverse link to previous node. They first of all check whether, valid route to destination is present or not. If, valid route is present then another condition must hold i.e intermediate node's sequence number should be at least as great as destination sequence number in RREQ packet. If both conditions hold, then that node generates RREP i.e. reply packet. If valid route is not present then RREQ is further forwarded. As RREQ is forwarded, hop count is incremented. While sending RREQ, 
intermediate nodes start a timer. If reply doesn't come within that time means, there is no more active route or link failure has occurred [11].

RREP contains IP address of source as well as destination, and destination sequence number. Once the node creates the forward route entry, it forwards the RREP to the destination node. The RREP is thus forwarded hop by hop to the source node, as indicated in Figure 2. Once the source receives the RREP, it can utilize the path for the transmission of data packets [4].

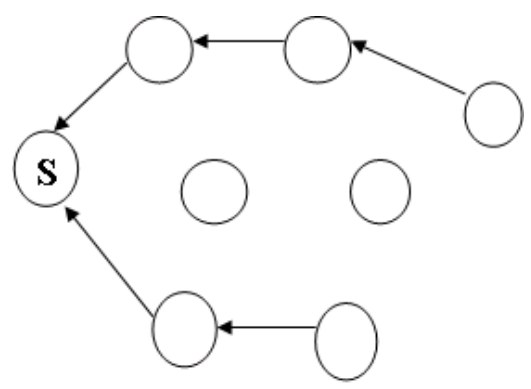

Fig 2: RREP Propagation [17].

\subsection{AODV Route Maintenance}

As MANET is dynamic i.e. mobility and topology of nodes always change, link break occurs. When path breaks, both the nodes inform their end nodes about link failure, who were using that path by sending RERR i.e. error message as illustrated in Figure 3. End nodes delete their entries from route table, as path is no longer useful. If source node still wants to communicate with destination, it reinitiates RREQ broadcasting or path finding process or repair broken link [11].

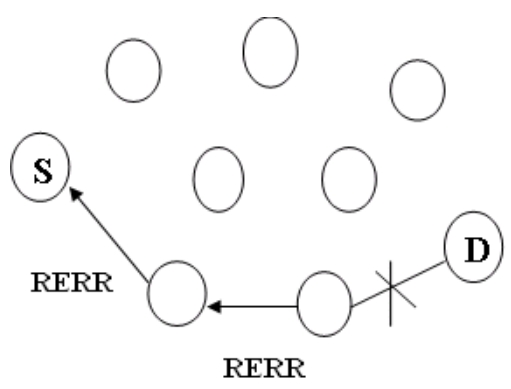

Fig 3: RERR Message [17].

\subsection{Problem Statement}

In the conventional AODV routing mechanism, a node broadcasts or floods RREQ message to its neighbours when it wants to communicate with a destination node [18]. If intermediate nodes' lifetime is less, that node expires after some time. Thus, it may not be able to forward the RREP message on the reverse path. Hence, the source node would have to rebroadcast the RREQ message in order to find a path for communicating to the destination node. This may cause congestion in the network, decrease the packet delivery ratio; increase the end to end delay and unnecessary rebroadcasting of RREQ packets [19].

\section{IMPLEMENTATION OF THE SYSTEM}

Proposed solution for this problem is given by OAODV which is based on AODV.

\subsection{Optimized Ad Hoc on Demand Routing Protocol (OAODV)}

Each node has a certain battery life and node density in its surrounding which is saved in the routing table of proposed OAODV protocol [14]. The intermediate node doesn't forward the RREQ message immediately if there is a route to destination. In fact, it will first check its lifetime and calculate the node density of its surrounding. Second parameter is taken into consideration because; there should be sufficient number of nodes to forward RREQ. Hello messages are used to determine neighbour connectivity or node density [20]. Two thresholds are introduced say ThB for RREQ rebroadcasting and $\mathrm{ThN}$ for node density of the environment. If the battery life and node density of the intermediate node, who receives the RREQ is greater than ThB and ThN, it can be concluded that, the broadcast of RREQ successfully reaches the destination node and so the intermediate node can rebroadcast RREQ message. If the ratio is less than $\mathrm{ThB}$ and $\mathrm{ThN}$, the intermediate node buffers the packets and repeats the above process iteratively until either the broadcast is successful or the number of attempts exceeds a threshold. This process helps to decrease unnecessary packet rebroadcasting and increase the throughput [12].

\subsection{Algorithm}

Algorithm for OAODV broadcast mechanism: \{

Begin

//Initialization of routing parameters like sequence number, threshold, MAC parameters, etc.

User requests for data transmission.

Case: Node is not the destination and has no path to the destination;

Repeat the loop till numbers of tries are less than maximum number of RREQ retries.

\{

Increment tries by one;

Check own battery life;

Calculate the threshold with the help of statistics i.e. Brute Force method;

Calculate the node density of the surrounding;

If Battery life is less than the Threshold (ThB) Remain silent, drop RREQ.

Elself count is less than the Threshold (ThN)

Packets are stored in node's buffer; else

RREQ is broadcasted further.

\}

//End of algorithm

\section{RESULTS AND PERFORMANCE ANALYSIS}

Network Simulator with 2.34 version is used on Ubuntu 10.10 operating system for the simulations. As mentioned earlier, study of AODV and optimized AODV protocol (OAODV) performed. 


\subsection{Simulation Environment}

The IEEE 802.11 Medium Access Control (MAC) Distributed Coordination Function (DCF) protocol is used in simulations [21]. Following are the simulation parameters.

- Range transmission : 300 meter

- Carrier sensing range : 300 meter

- Simulation time : $120 \mathrm{sec}$

- Number of nodes : 10, 20, 30, 40, 50

- Topology area : $500 \mathrm{~m}$ x $500 \mathrm{~m}$

- Mobility model : Random way point

- Traffic type : TCP

- Maximum speed : $10 \mathrm{~m} / \mathrm{s}$

- Packet size : 512 bytes for TCP

- Initial energy : 60 Joules

- $\quad$ Pause time : $2 \mathrm{sec}$

- Type of antenna : Omni directional

- Channel type : Wireless channel
- Maximum packets in queue : 50

- $\quad$ Radio propagation model : Two ray ground

- Network interface type : WirelessPhy

- Interface queue type : Drop tail / PriQueue

\subsection{Evaluation Metrics}

\subsubsection{Battery Lifetime}

Remaining energy is calculated as shown in equation (1).

Remaining energy = Initial energy - Energy used

There is a reduction in energy consumption of network for OAODV as compared to AODV because each node is now aware of its energy constraints for data communication as shown in Figure 4, Figure 5, Figure 6, Figure 7 and Figure 8.

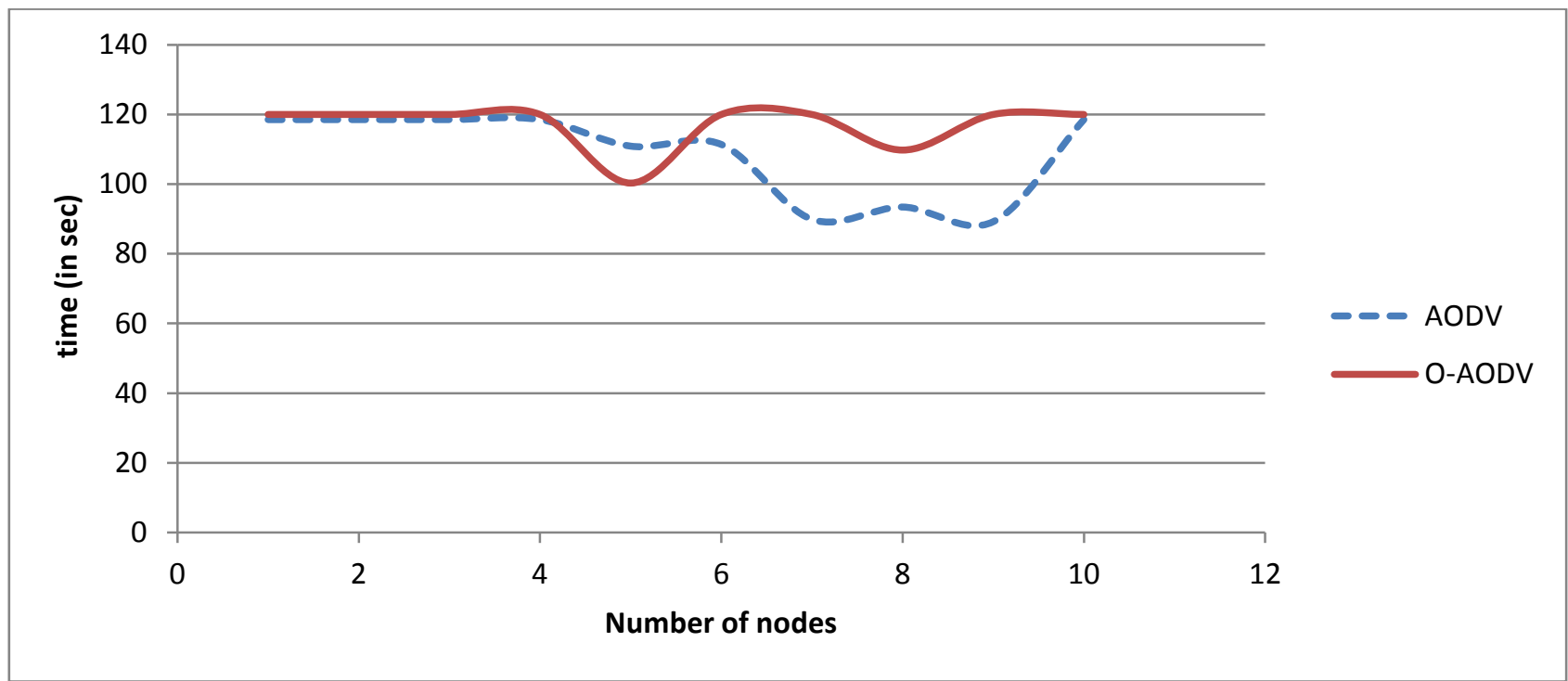

Fig 4: Comparison of AODV and Optimized AODV in terms of battery lifetime for TCP traffic for ten nodes.

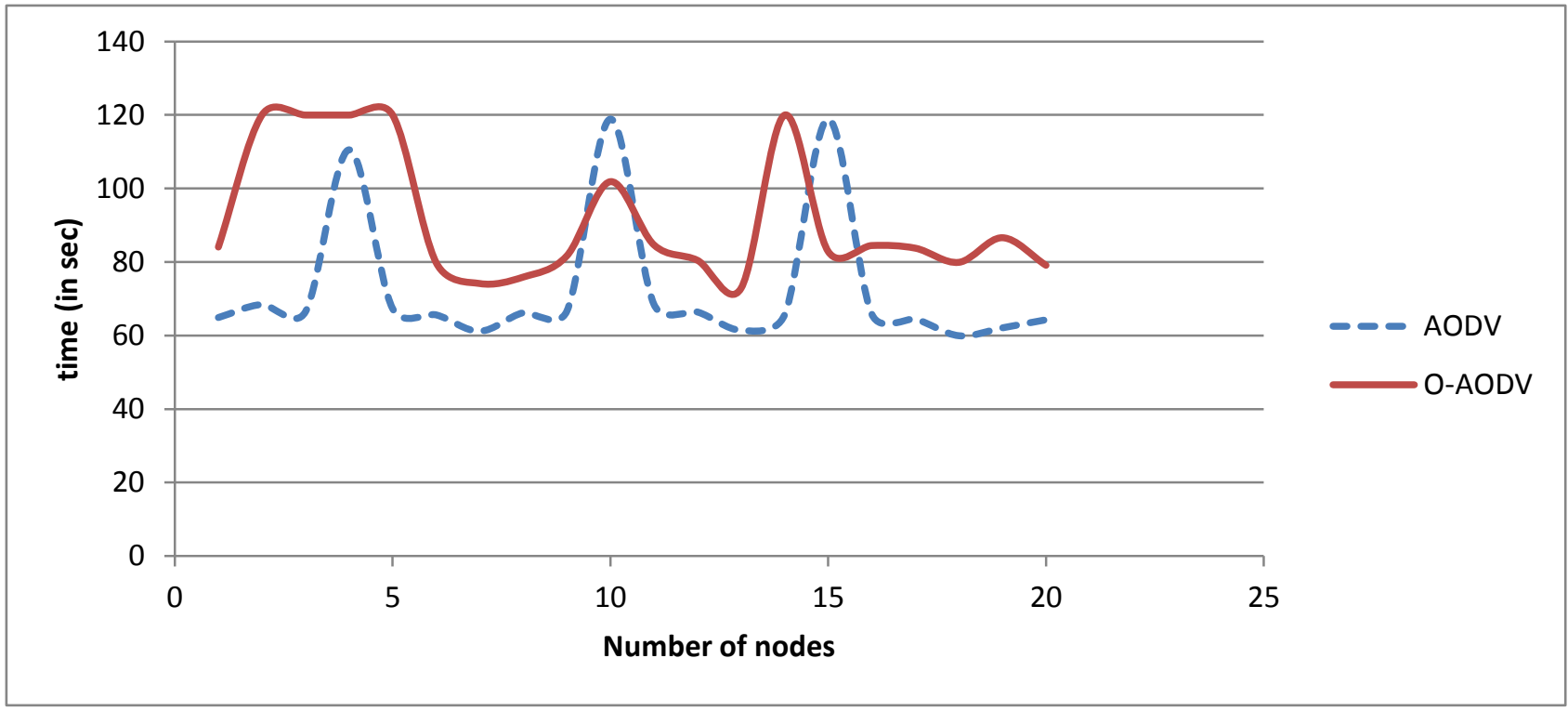

Fig 5: Comparison of AODV and Optimized AODV in terms of battery lifetime for TCP traffic for twenty nodes. 


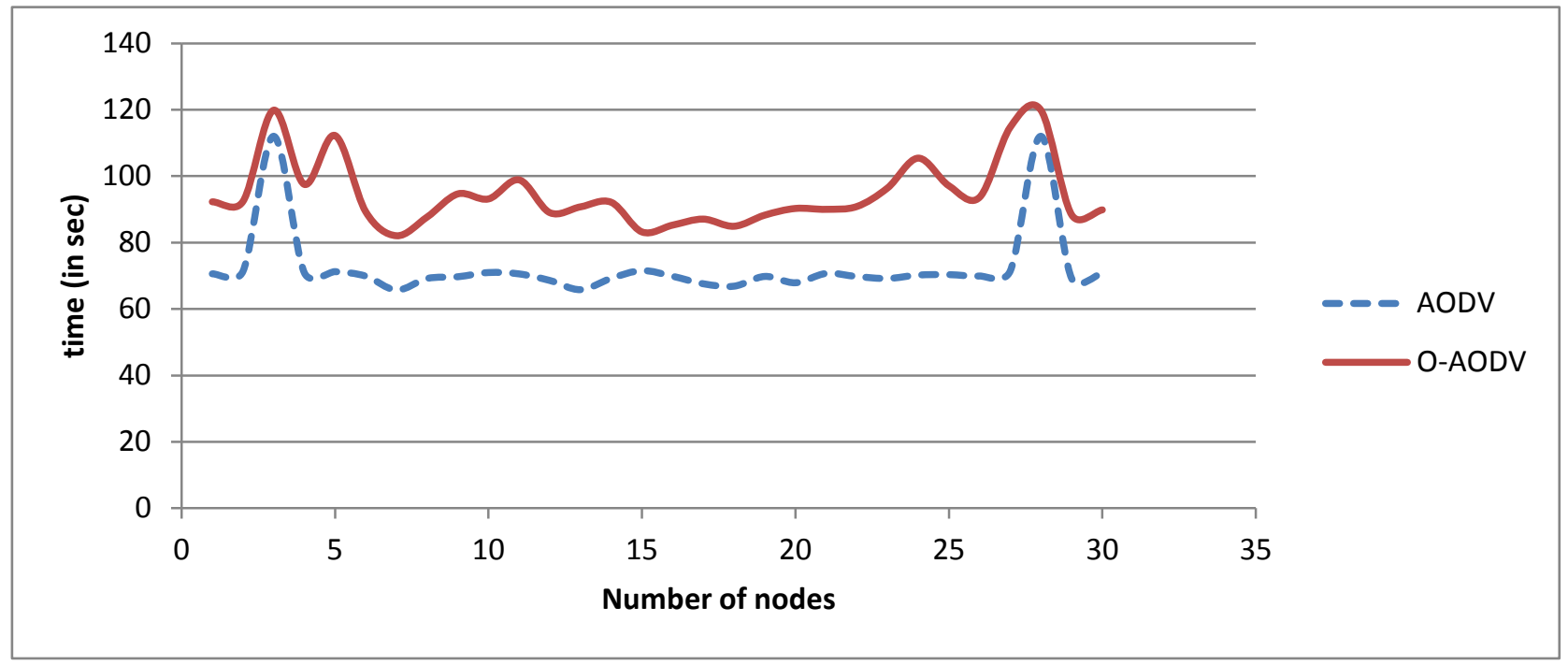

Fig 6: Comparison of AODV and Optimized AODV in terms of battery lifetime for TCP traffic for thirty nodes.

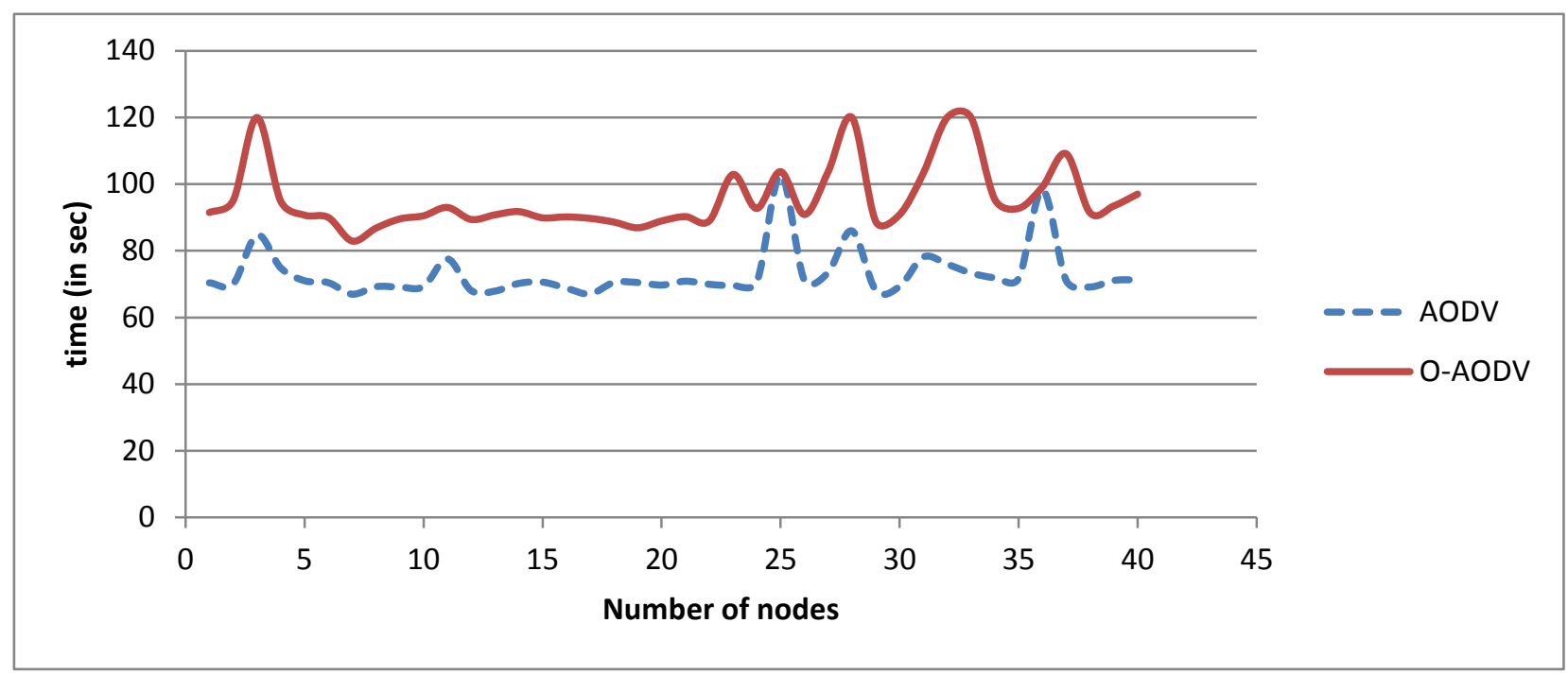

Fig 7: Comparison of AODV and Optimized AODV in terms of battery lifetime for TCP traffic for fourty nodes.

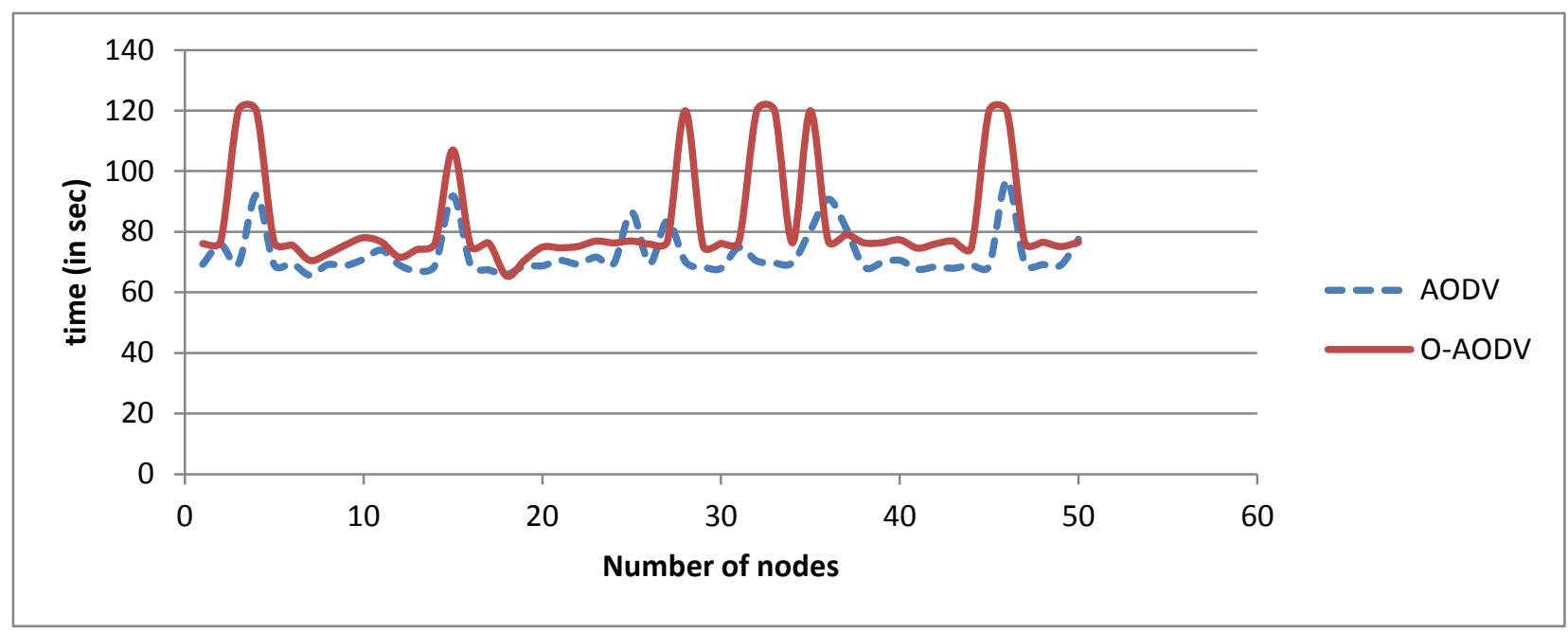

Fig 8: Comparison of AODV and Optimized AODV in terms of battery lifetime for TCP traffic for fifty nodes. 


\subsubsection{Average Throughput}

Analysis on various scenarios based on different number of nodes is performed. As compared to the existing AODV, proposed schemes in forwarding a route request are more effective in reducing the flooding overhead and efficient network lifetime as well as throughput thereby, decreasing the network latency. Simulation results indicate that, the proposed scheme provides enhanced performance. OAODV generates good throughput as compared to AODV as shown in Figure 9, Figure 10, Figure 11, Figure 12 and Figure 13. Throughput is calculated as shown in equation (2).

Throughput $=\frac{\text { Number of packets successfully received }}{\text { Receive time-Send time }}$

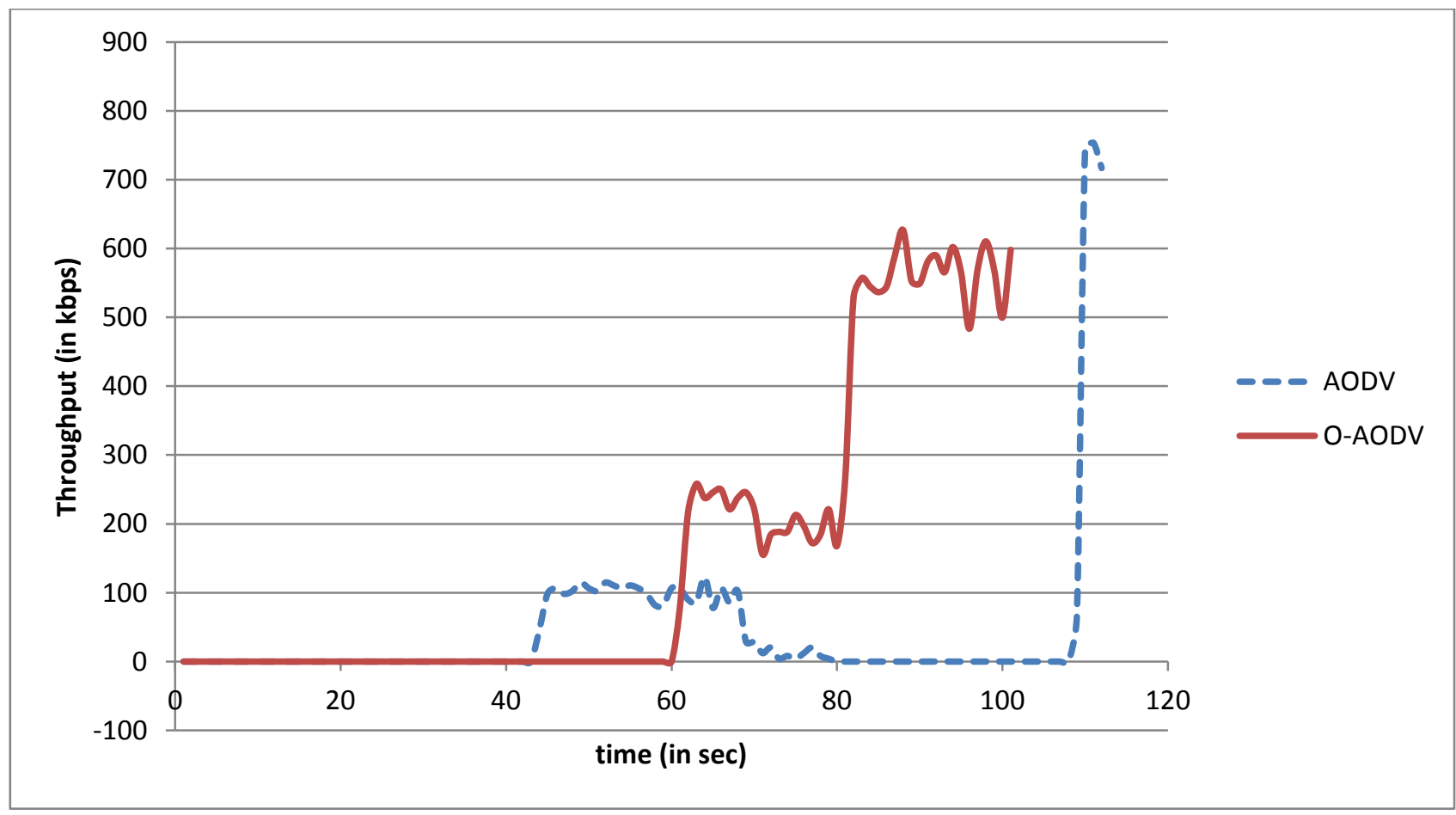

Fig 9: Comparison of AODV and Optimized AODV in terms of throughput for TCP traffic for ten nodes.

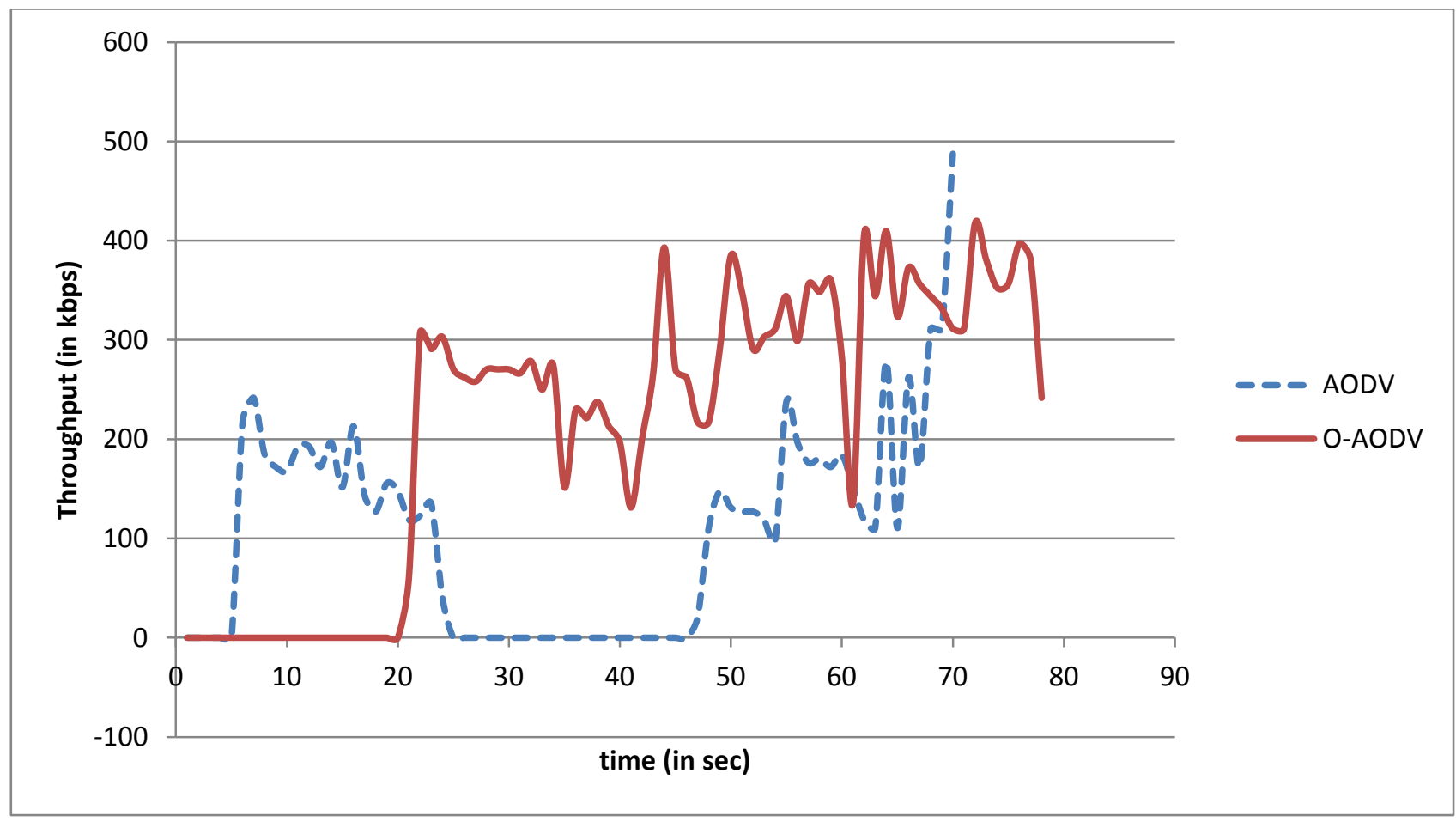

Fig 10: Comparison of AODV and Optimized AODV in terms of throughput for TCP traffic for twenty nodes. 


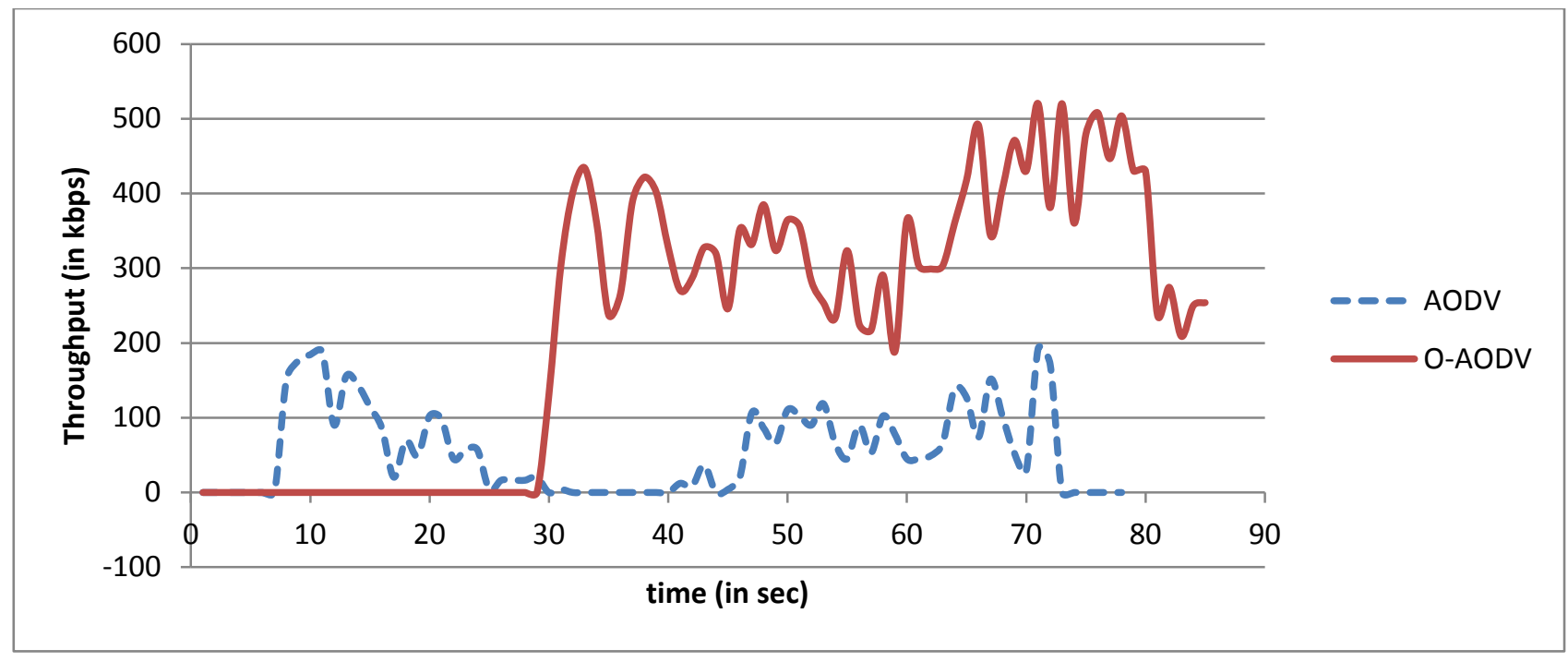

Fig 11: Comparison of AODV and Optimized AODV in terms of throughput for TCP traffic for thirty nodes.

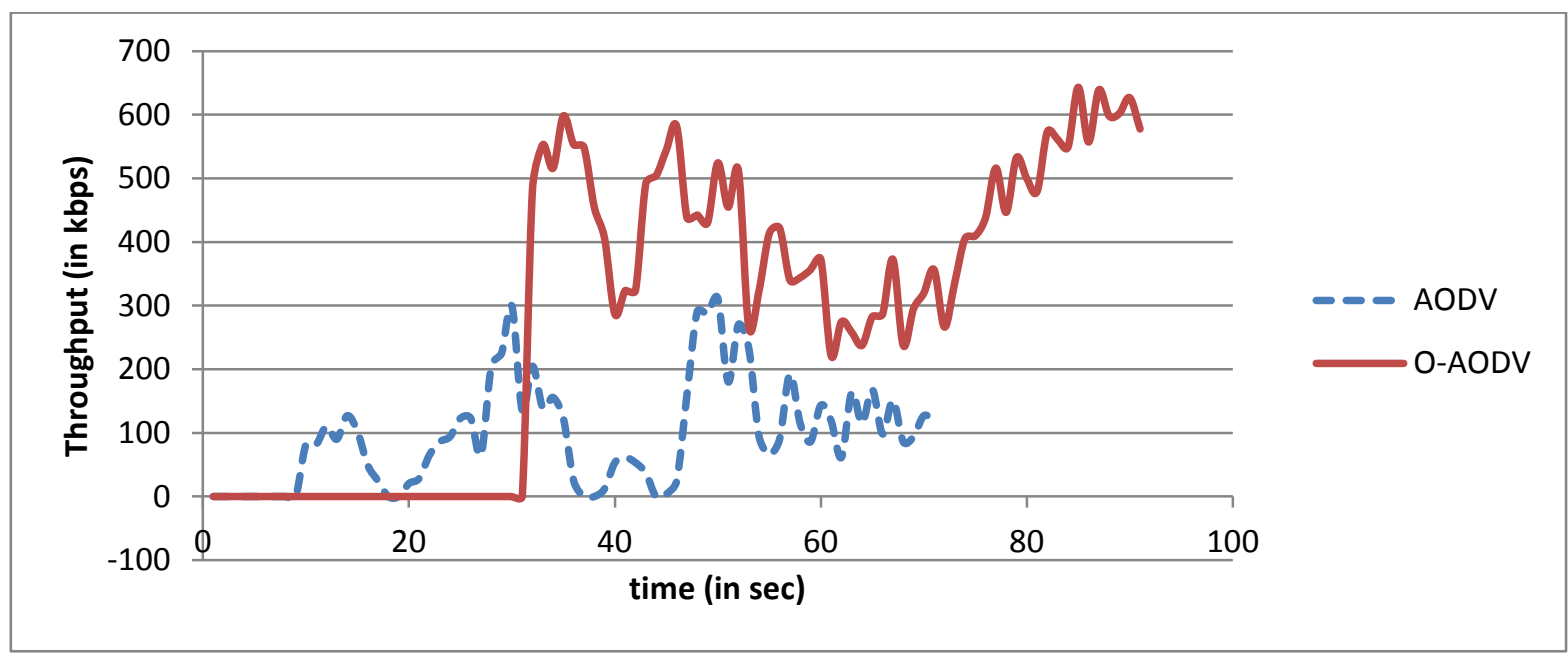

Fig 12: Comparison of AODV and Optimized AODV in terms of throughput for TCP traffic for fourty nodes.

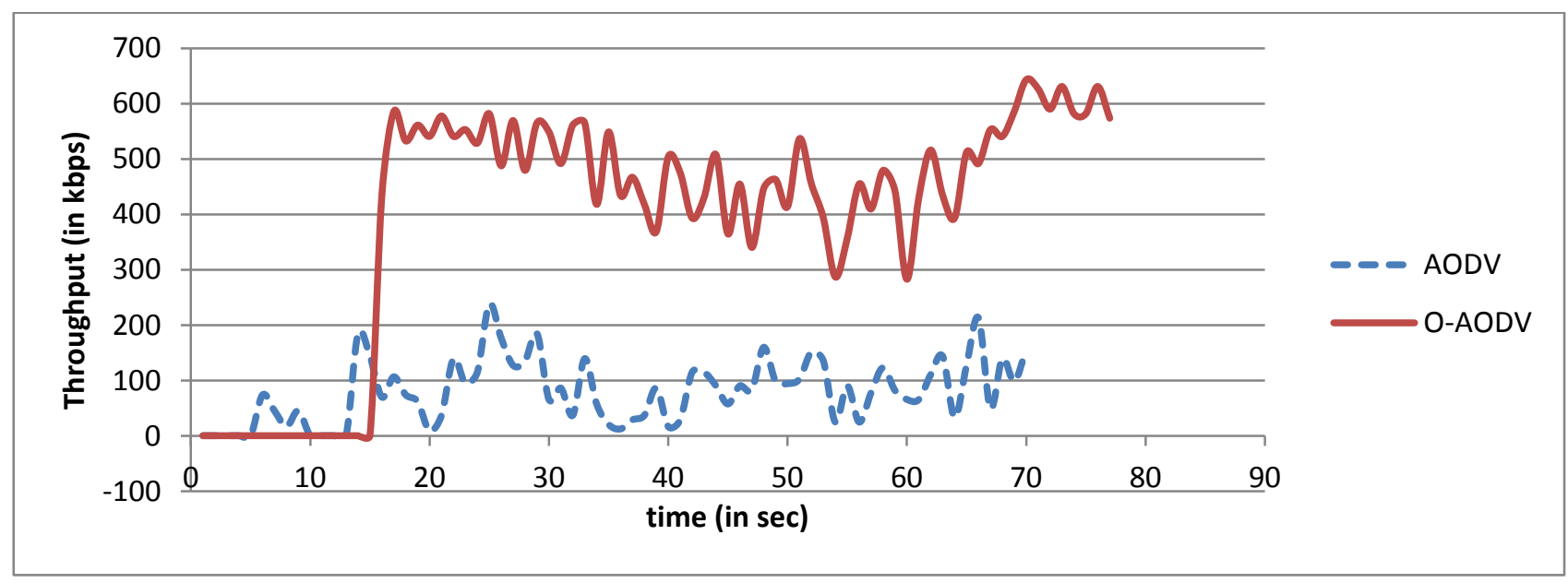

Fig 13: Comparison of AODV and Optimized AODV in terms of throughput for TCP traffic for fifty nodes. 


\section{CONCLUSION AND FUTURE SCOPE}

This paper discusses how energy is one of the important factor for MANET. Energy efficient AODV routing protocol is proposed. This paper presented an Optimized Mobile Ad Hoc Network on Demand routing protocol, which modifies broadcast mechanism of conventional AODV routing protocol. Successful delivery of RREP is important in MANET. If reply is lost, new rote discovery process has to be reinitiated. OAODV avoids unnecessary broadcasting of RREQ information. In this proposal, the node does not broadcast the routing request (RREQ) if it does not have sufficient energy (battery lifetime), and until the node density in its surrounding exceeds a particular threshold. After comparing AODV with OAODV in terms of battery lifetime and throughput, it is observed that the new protocol is much better than AODV and lengthens the battery lifetime.

In future, there is a need to find the effect of this algorithm for different mobility models.

\section{REFERENCES}

[1] Saleh Ali K. Al Omari and Putra Sumari, " An overview of Mobile Ad Hoc Networks for the existing protocols and applications", International Journal on applications of graph theory in wireless ad hoc networks and sensor networks, vol. 2, no. 1, March 2010.

[2] Divya Sharma and Ashwani Kush, "Performance comparison of energy efficient AODV protocols", International Journal of Computing and Business Research (IJCBR), vol. 2, Issue 1, 2011.

[3] Annapurna P Patil, Dr. K. Rajani kanth, Bathey Sharanya, M. P. Dinesh Kumar and J. Malavika, "Design of an energy efficient routing protocol for MANETs based on AODV", IJCSI International Journal of Computer Science Issues, vol. 8, no. 1, Issue 4, July 2011.

[4] N. Sumathi and Dr. Antony Selvadoss Thanamani, "Evaluation of energy efficient reactive routing protocols in QoS enabled routing for MANETs", International Journal of Computer Applications, vol. 14, no. 2, January 2011.

[5] Abdusy Syarif and Riri Fitri Sari, "Performance analysis of AODV-UI routing protocol with energy consumption improvement under mobility models in hybrid ad hoc network", International Journal on Computer Science and Engineering, vol. 3, no. 7, July 2011.

[6] Jochen Schiller, "Mobile Communications", Pearson Publication, $2^{\text {nd }}$ Edition, 2005.

[7] Parma Nand and Dr. S. C. Sharma, "Performance study of broadcast based mobile ad hoc routing protocols AODV, DSR and DYMO”, International Journal of Security and Its Applications, vol. 5, no. 1, January 2011.

[8] Charles E. Perkins and Pravin Bhagwat, "Highly Dynamic Destination Sequenced Distance Vector Routing (DSDV) for mobile computers", Proc. ACM SICOMM 94, London, UK, October 1994.

[9] David Johnson and David Maltz, "Dynamic Source Routing in ad hoc wireless networks", Computer
Communications Review - Proceedings of SICOMM, August 1996.

[10] Dr. Sanjay Sharma and Pushpinder Singh Patheja, "Improving AODV routing protocol with Priority and Power Efficiency in Mobile Ad hoc WiMAX Network", International Journal of Computer Technology and Electronics Engineering (IJCTEE), pp. 87-93, vol. 2, Issue 1, February 2012.

[11] C. E. Perkins, E. M. Royer and S. Das, "Ad hoc On Demand Distance Vector (AODV) routing", IETF Internet draft, draft-ietf-manet-aodv-08.xt, March 2001.

[12] K. Murugan and S. Shanmugavel, "Traffic Dependent and Energy Based Time Delay Routing Algorithms for improving energy efficiency in Mobile Ad Hoc Networks", EURASIP Journal on Wireless Communications and Networking, pp. 625-634, 2005.

[13] Thriveni Ja, Anita Kanavallia, K. R. Venugopala and L. M. Patnaik, "Probabilistic Mean Energy Flooding to increase the survivability of MANET", Proceedings of the International MultiConference of Engineers and Computer Scientists, pp. 19-21, vol. 2, Hong Kong, March 2008.

[14] Zhang Jianwu, Zou Jingyuan and Zhao Qi, "MANET routing protocol for improving routing discovery based on AODV", International Conference on Networks Security, Wireless Communications and Trusted Computing, Telecommunication School, Hangzhou Dianzi University, vol. 2, China, 2009. (DOI 10.1109/NSWCTC.2009.11).

[15] Sunil Taneja, Ashwani Kush, Amandeep Makkar, and Bharat Bhushand, "Power management in Mobile Ad hoc Network", Journal of Computing and Business Research (IJCBR), vol. 2, Issue 1, 2011.

[16] C. Perkins, E. Belding Royer and S. Das, "Ad hoc on Demand Distance Vector (AODV) routing", Network Working Group, RFC-3561, July 2003.

[17] Srinivasan Keshav, "An Engineering Approach to Computer Networking, ATM Network, the Internet and the Telephone Network", Pearson Education.

[18] Venetis Kanakaris, David Ndzi and Kyriakos Ovaliadis, "Improving AODV performance using Dynamic Density Driven Route Request Forwarding", International Journal of Wireless \& Mobile Networks, vol. 3, no. 3, June 2011. (DOI: 10.5121/ijwmn.2011.3302)

[19] Mahesh Kumar Yadav, Ram Kishan Khola and Deepak Dembla, "Modeling, analysis and implementation of improved AODV routing protocol in MANETs", International Journal of Computer Applications, vol. 41, no. 21, March 2012.

[20] Jaya Jacob and V. Seethalakshmi, "Performance analysis and enhancement of routing protocol in MANET", International Journal of Modern Engineering Research, pp. 323-328, vol. 2, Issue 2, March - April 2012.

[21] IEEE standard for Wireless LAN Medium Access Control (MAC) and Physical Layer (PHY) specifications, ISO/IEC 8802-11: 1999(E) (1999). 